\title{
Frictional Effects on Gear Tooth Contact Analysis
}

\author{
Zheng Li and Ken Mao \\ The Gearbox Research Institute, Dalian Huarui Heavy Group Co., Ltd., School of Engineering, Warwick University, \\ Coventry CV4 7AL, UK \\ Correspondence should be addressed to Ken Mao; k.mao@warwick.co.uk
}

Received 16 February 2013; Accepted 20 June 2013

Academic Editor: Patrick De Baets

Copyright (C) $2013 \mathrm{Z}$. Li and K. Mao. This is an open access article distributed under the Creative Commons Attribution License, which permits unrestricted use, distribution, and reproduction in any medium, provided the original work is properly cited.

The present paper concentrates on the investigations regarding the situations of frictional shear stress of gear teeth and the relevant frictional effects on bending stresses and transmission error in gear meshing. Sliding friction is one of the major reasons causing gear failure and vibration; the adequate consideration of frictional effects is essential for understanding gear contact behavior accurately. An analysis of tooth frictional effect on gear performance in spur gear is presented using finite element method. Nonlinear finite element model for gear tooth contact with rolling/sliding is then developed. The contact zones for multiple tooth pairs are identified and the associated integration situation is derived. The illustrated bending stress and transmission error results with static and dynamic boundary conditions indicate the significant effects due to the sliding friction between the surfaces of contacted gear teeth, and the friction effect can not be ignored. To understand the particular static and dynamic frictional effects on gear tooth contact analysis, some significant phenomena of gained results will also be discussed. The potentially significant contribution of tooth frictional shear stress is presented, particularly in the case of gear tooth contact analysis with both static and dynamic boundary conditions.

\section{Introduction}

In modern world, many reliable gear manufacture techniques have existed and operated for modern industry; the advanced high performance gears products are indispensable components for lots of power-train transmission systems. As a basic transmission part, gears have been used for three thousand years in history, and the investigation of gear design techniques and product performance kept developing all the time. In 1892, Wilfred Lewis first presented a formula for evaluating the bending stress of gear teeth in which the tooth form entered into the equation. The formula still remains the basis for most gear design today, and the parameter proposed as "Lewis stress" can indicate the bending stress of loaded contacted gear teeth accurately. But in further investigation on the basis of the application of Lewis stress, the nonlinear responses due to frictional effects in meshing process have become obvious gradually, and many scientists have been tried to investigate any reliable techniques to predict frictional effect on gear performance. In 1982, Barakat [1] presented a technique for the determination of the instantaneous coefficient of friction at gear tooth contact by considering tooth fillet strains using finite element method. Ishibashi et al. [2] researched the friction situation of contacted teeth at start of running in 1987, and they considered lubricant conditions in gear meshing and found that reduction in the tooth surface roughness is very effective to decrease the coefficient of friction and to prevent the cold scoring which might become the origins of severe failure in the lengthy running. The primary investigations of frictional effects were just focused on how to determine the coefficient of friction or the particular amount of friction in gear meshing, but the significant frictional effects on gear static and dynamic performance were still secrets.

In 1990s, some gear tooth contact analysis started to consider frictional effects in the investigations; it really made very significant progress of gear performance investigation, and the presented technologies regarding predicting the linear and nonlinear responses to gear tooth contact were more accurate and reliable during this period. In 1994, Vijayarangan and Ganesan [3] analyzed static contact stress of spur gear tooth with frictional effect using finite element method and Lagrangian multiplier technique. They discussed the actual length of contact against the calculated length of 
contact and presented the variation of contact stress along the contact surface in a direction normal to the mating surfaces. In 1996, Rebbechi et al. [4] did research which was focused on dynamic friction status; they measured the strain at the root fillets of two successive teeth, and the results of their research demonstrated that the friction coefficient does not appear to be significantly influenced by the sliding reversal at the pitch point and that the values of coefficient of friction found are in accord with those in general use. The coefficient of friction was found to increase at low sliding speed. The investigation conclusions of this period were much more reliable than last decade, but due to the lack of high precision tools (computer software and experiment equipment), the accuracy of results was still limited; the evaluation of new findings usually used empirical results as measurement.

Nowadays, many software tools with perfect precision have been developed and kept updating; the researches of gear performance and frictional effects have become more convenient and accurate with the help of various finite element simulation software tools. In 2002, Velex and Sainsot [5] presented an analytical analysis of tooth friction excitations in errorless spur and helical gears base on Coulomb model. Just for the most typical spur gear, Mihalidis et al. [6] calculated the coefficient of friction at several contact points along the path of contact based on a mixed lubrication model in the same year; their production was very significant for following deeper research of gear contact friction. In recent years, some new conclusions have been presented, and the frictional effects status and nonlinear performances caused by such slide friction have been paid more attention by gear designers. In 2007, Kim and Singh [7] presented a new gear surface roughness induced noise source model while taking into account the sliding contacts between meshing gear teeth, and they considered dynamic condition for predicting transmission noise. He et al. [8] proposed a new dynamic contacted model which considered sliding friction; the model is highly accurate enough and significant for gear performance analyzing. The nonlinear dynamic responses and frictional effects are really complicated problems of gear research, especially the relationship between individual dynamic response (noise and vibration) and frictional effects, and the conclusions will build significant basis for further relevant investigations.

According to empirical conception foundations and the literature, this paper will analyze the frictional effects on gear tooth bending stress and transmission error in meshing using finite element method and attempt to explain the phenomena. For dynamic response, the transmission error is the main reason of noise and vibration of system and can be affected by sliding friction obviously. The reliable microgeometry modification scheme is effective and necessary to decrease harmful noise and vibration, but the frictional effect is still available for transmission error regulation.

\section{Tooth Profile Generation and FEM Model}

The tooth profile of model was generated by mathematical method; the principle of generation is calculating sufficient

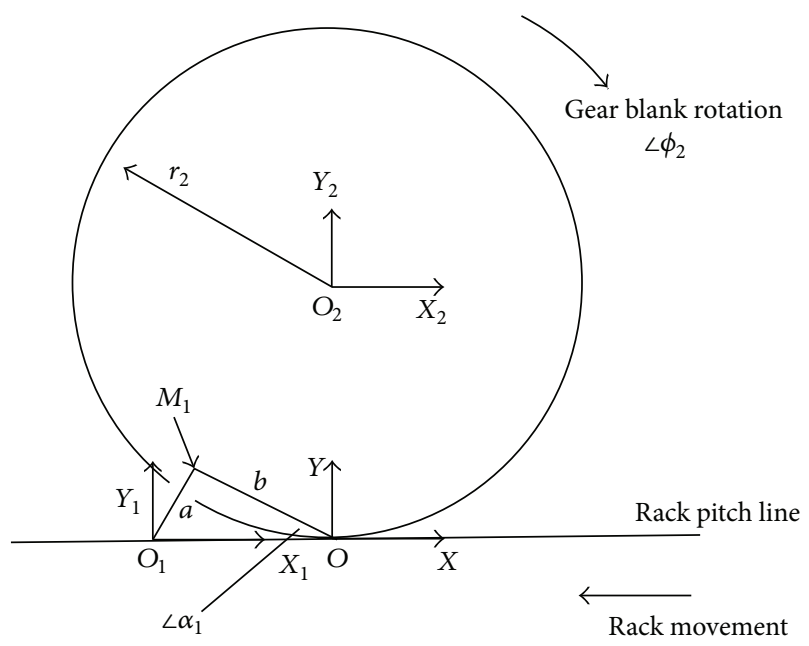

Figure 1: Involute profile generation. $\alpha_{1}$ : pressure angle, $\phi_{2}$ : gear rotation angle corresponding to rack movement of $b_{1}$.

point coordinates which belong in tooth profile and then connecting all of the points by straight lines. The whole processes can be divided into two parts: involute profile generation and root curve generation.

2.1. Involute Profile Generation. According to Figure 1, the equation for $\mathrm{O}_{1} a$ line is

$$
y_{1}=\frac{x_{1}}{\tan \alpha_{1}}
$$

If a point $M_{1}$ in $O_{1} a$ line will contact with the gear, the rack needs to be moved a distance $b_{1}\left(O_{1} O\right)$ to achieve the normal of point $M_{1}$ cross with point $O$ according to the gear conjugating theory:

$$
b_{1}=\frac{y_{1}}{\tan \alpha_{1}}+x_{1} .
$$

For coordinate transfer from $O_{1} X_{1} Y_{1}$ to $O X Y$,

$$
\begin{gathered}
x=x_{1}-b_{1}, \\
y=y_{1} .
\end{gathered}
$$

Then for coordinate transfer from $O X Y$ to $\mathrm{O}_{2} \mathrm{X}_{2} Y_{2}$,

(i) transfer

$$
\begin{gathered}
x^{\prime}=x, \\
y^{\prime}=y-r_{2},
\end{gathered}
$$

(ii) rotate

$$
\begin{aligned}
& x_{2}=x^{\prime} \cos \phi_{2}-y^{\prime} \sin \phi_{2} \text {, } \\
& y_{2}=x^{\prime} \sin \phi_{2}+y^{\prime} \cos \phi_{2} \text {, } \\
& \begin{aligned}
x_{2} & =x \cos \phi_{2}-\left(y-r_{2}\right) \sin \phi_{2}, \\
y_{2} & =x \sin \phi_{2}+\left(y-r_{2}\right) \cos \phi_{2},
\end{aligned} \\
& \Longrightarrow \begin{array}{l}
x_{2}=x \cos \phi_{2}-y \sin \phi_{2}+r_{2} \sin \phi_{2}, \\
y_{2}=x \sin \phi_{2}+y \cos \phi_{2}-r_{2} \cos \phi_{2}
\end{array}
\end{aligned}
$$




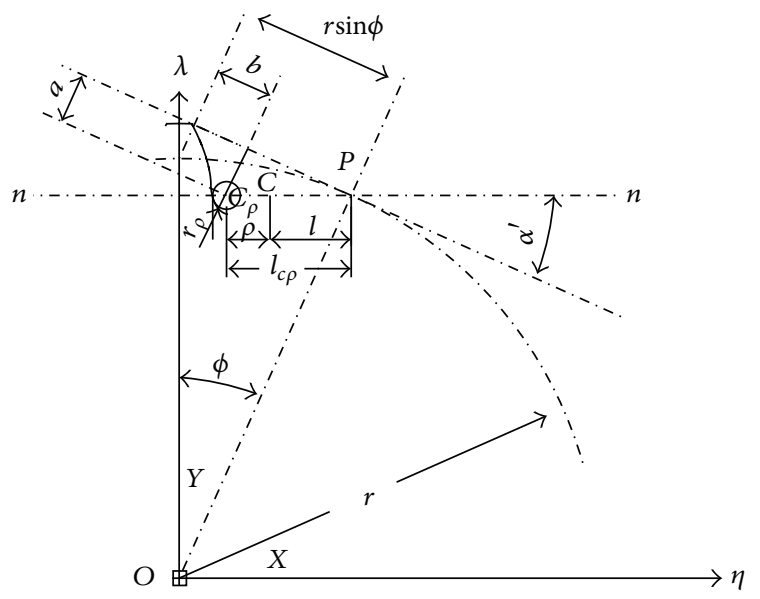

FIGURE 2: Root profile generation.

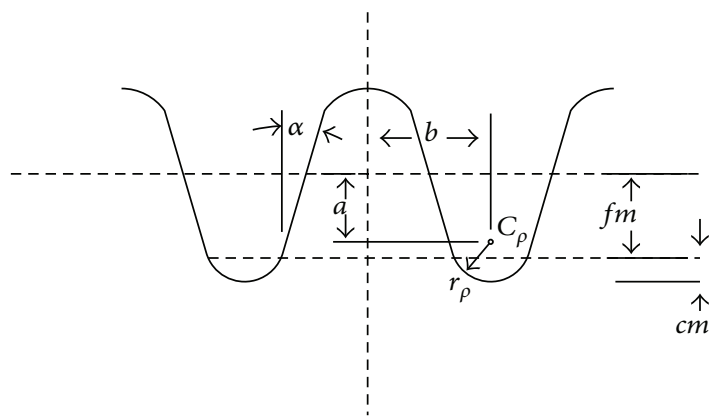

FIgURE 3: The hob tooth profile.

Therefore, the point coordinates in system $\mathrm{O}_{2} \mathrm{X}_{2} Y_{2}$ can be written as following equations:

$$
\begin{aligned}
& x_{2}=\left(x_{1}-b_{1}\right) \cos \phi_{2}-\left(y_{1}-r_{2}\right) \sin \phi_{2}, \\
& y_{2}=\left(x_{1}-b_{1}\right) \sin \phi_{2}+\left(y_{1}-r_{2}\right) \cos \phi_{2},
\end{aligned}
$$

where $r_{2}$ is the gear pitch circle radius, $\phi_{2}=b_{1} / r_{2}$.

The gear profile generated by line $O_{1} a$ will be obtained by solving the combined equations (1), (2), and (6); the final simultaneous equations are

$$
\begin{gathered}
y_{1}=\frac{x_{1}}{\tan \alpha_{1}}, \\
b_{1}=\frac{y_{1}}{\tan \alpha_{1}}+x_{1}, \\
x_{2}=\left(x_{1}-b_{1}\right) \cos \phi_{2}-\left(y_{1}-r_{2}\right) \sin \phi_{2}, \\
y_{2}=\left(x_{1}-b_{1}\right) \sin \phi_{2}+\left(y_{1}-r_{2}\right) \cos \phi_{2} .
\end{gathered}
$$

2.2. Root Curve Generation. Global coordinate system: $O \eta \lambda$. The final simultaneous equations are expressed as following, and the meanings of necessary factors are indicated by Figures 2 and 3.

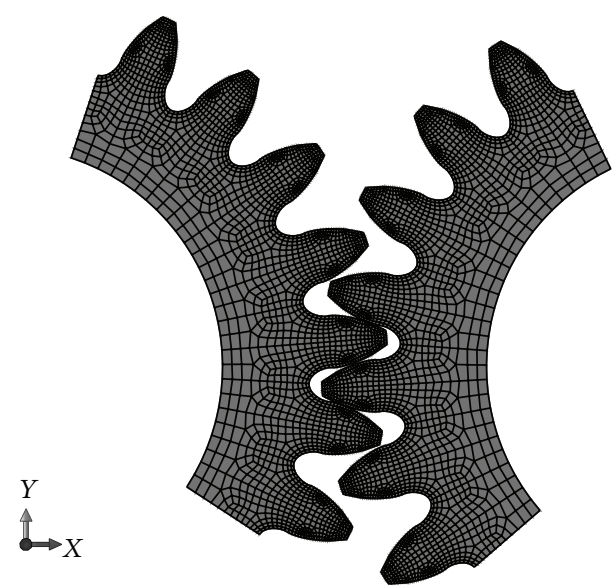

Figure 4: Gear geometry and contact model.

The final simultaneous equations are

$$
\begin{gathered}
a=f m+c m-r_{\rho}, \\
b=\frac{\pi m}{2}, \\
r_{\rho}=\frac{c m}{1-\sin \alpha}, \\
\eta=r \sin \phi-\left(\frac{a}{\sin \alpha^{\prime}}+r_{\rho}\right) \cos \left(\alpha^{\prime}-\phi\right), \\
\lambda=r \cos \phi-\left(\frac{a}{\sin \alpha^{\prime}}+r_{\rho}\right) \sin \left(\alpha^{\prime}-\phi\right) . \\
\sin \phi=\frac{1}{r}\left(\frac{a}{\tan \alpha^{\prime}}+b\right),
\end{gathered}
$$

where $m$ is module, $f$ is depth coefficient: whole depth/ module, $c$ is Clearance coefficient, $\alpha$ : pressure angle, $\alpha^{\prime}$ is pariable amount, the variable zone is $0 \sim 90^{\circ}$, and $(\eta, \lambda)$ is required point coordinate.

In this research, a two-dimension finite element model is built using ABAQUS to simulate standard involute spur gear pair. The gear will run at $100 \mathrm{rad} / \mathrm{s}$ with $30 \mathrm{Nm}$ load. The primary parameters of the gears are listed in Table 1. Figure 4 shows the 2D gear pair model for the study.

\section{Static Bending Stress and Transmission Error}

Bending stress analysis is an important objective of static performance investigation because it is the primary reason of various gear tooth failures. The empirical conceptions of static gear stress analysis have already demonstrated that the gear tooth can be considered as cantilever beam, so the complicated contact question can be simplified to solve a simple cantilever beam strength calculation. The examinations of bending stress will consult Lewis' stress theories. According to the common cantilever beams hypothesis for gear research, the Lewis stress formula can be obtained from the most basic theories regarding cantilever beam. 
TABLE 1: Gear model parameters.

\begin{tabular}{lc}
\hline Type & Spur \\
Module & $2 \mathrm{~mm}$ \\
Number of teeth & 24 \\
Pressure angle & $20^{\circ}$ \\
Tooth width & $3.142 \mathrm{~mm}$ \\
Tip radius & $26 \mathrm{~mm}$ \\
Root radius & $21.5 \mathrm{~mm}$ \\
Modification factor & 0 \\
Contact ratio & 1.77 \\
Element size: & $0.2 \mathrm{~mm}$ \\
Material & \\
$\quad$ Steel & \\
$\quad$ Density & \\
$\quad$ Young's modulus & $7.8 \mathrm{~g} / \mathrm{cm}^{3}$ \\
$\quad$ Poisson's ratio & $210000 \mathrm{MPa}$ \\
\hline
\end{tabular}

Transmission error is considered to be an important excitation mechanism for gear noise and vibration, and many researches relate to gear noise and vibration are always focused on transmission error situation to achieve particular purposes. Transmission error has been defined as the difference between the actual position of the output gear and the position it would occupy if the gear drive were perfect. Actually, transmission error is an important kind of deformation for the gear set and it is the main source of noise and vibration because the deformation and friction will cause harmful impact and abrasion. In actual design and manufacture processes, the deformation and friction must exist, which means the transmission error cannot be eliminated. The frictional effect on gear meshing will also affect transmission error obviously, and it will make the transmission error anomalous to produce serious noise and vibration. The investigation of transmission error is very significant for optimizing dynamic performance by reducing noise and vibration because the most effective method to control harmful dynamic response is transmission error regulation.

In this research, the particular static bending tensile stress and transmission error results will be illustrated. The investigation analyzes two coefficients of friction, one with value 0 and the other with value 0.05 to determine the significant frictional effects on static bending tensile stress and transmission error.

Figure 5 is the result of gear static bending tensile stress with static boundary conditions group. The interaction points $\mathrm{A}$ and $\mathrm{B}$ are two special points which implicate certain significant contact events in gear meshing process.

The result lines indicate that the general trends of static bending tensile stresses with different coefficient of friction are very similar. Point $\mathrm{A}$ means that the gear tooth which the critical point belongs to is starting to contact at the moment; the interaction point $\mathrm{B}$ of the two lines indicates that there is no friction shear stress at the moment no matter how high the coefficient of friction of gear tooth surface is, and it implicates that the meshing gear teeth are mainly contacting

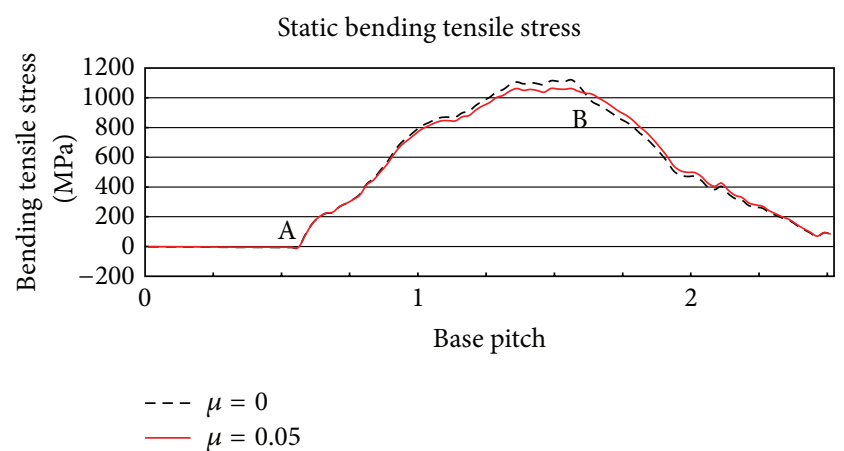

FIGURE 5: Static bending tensile stress results.

at pitch point in nature. In fact, a complete gear teeth contact process can be divided into two different contact phases: start point-pitch point and pitch point-finish point; the integrated bending stress status is different during these two contact phases due to the friction. It is also the most significant frictional effect on static bending stress.

According to both of the result lines in Figure 5, it can be seen that the relative positions of the two result lines are different. The position of line $\mu=0$ is higher than line $\mu=0.05$ during the segment between points A and B. It indicates that the bending stress with low coefficient of friction is higher in the first contact phase from start point to pitch point, but the status will be opposite in the second contact phase from pitch point to finish point. The implication of this phenomenon is that the friction can decrease bending tensile stress in the first contact phase but increase bending tensile stress in the second contact phase. The reason of this phenomenon can be explained by simple analysis of forces in gear meshing process as in the following discussions.

Whilst a pair of teeth starts to mesh, the input force will act on contact point with vertical direction to contact surface. The component of input force in vertical direction (relative to horizontal surface) is the bending stress which is the inspection object. If the friction between contact surfaces has been ignored, the force analysis of gear tooth should be shown in Figure 6 where $F_{n}$ is input force, $F_{t}$ and $F_{r}$ are vertical and horizontal components of $F_{n}$, and $O$ is contact point.

The force analysis bases on non-frictional situation of gear contact, but the friction must exist between contact surfaces due to relative sliding, and the bending stress will be affected by frictional effect. In frictional situation, the gear tooth contact process can be divided into two phases: start point-pitch point and pitch point-end point. It is necessary to notice that the pitch point is a special node; there is no friction force in the moment when the contact point overlaps pitch point, and the relative sliding direction will change from that moment. That means the friction force will start to reverse when the gear teeth are contacting at pitch point, and the bending stress status is different whilst the contact point stays before and after pitch point. According to the studies, the force analysis with frictional effect can be shown as in Figures 6(b) and 6(c). 


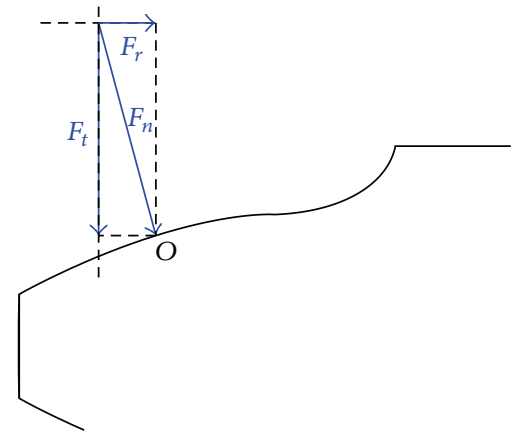

(a) Frictionless

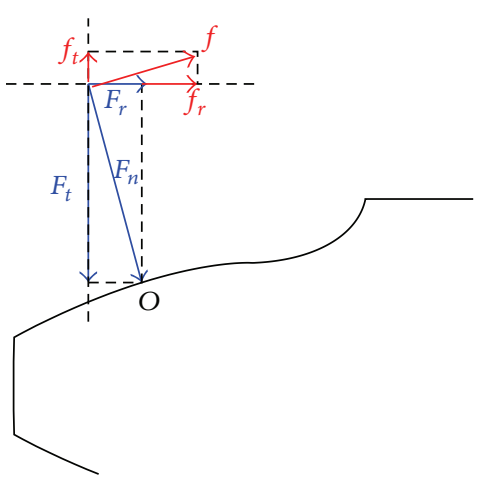

(b) The approach process

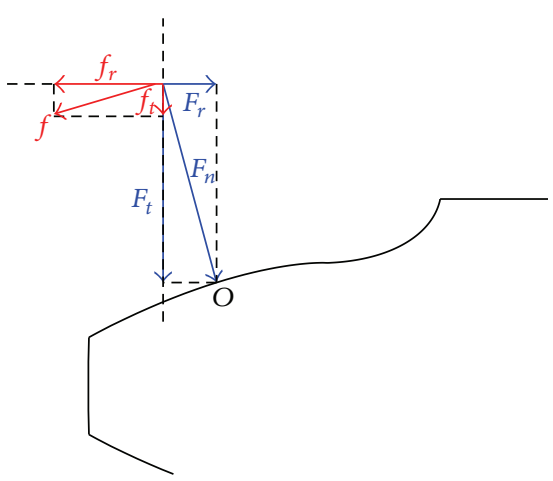

(c) The recess process

FIGURE 6: Gear contact force.

Figure 6 indicates the relation of the input force and friction force. The input force vertical component $F_{v}$ and horizontal component $F_{h}$ which cause bending stress of nonfrictional model should be

$$
\begin{aligned}
& F_{v}=F_{t}, \\
& F_{h}=F_{r} .
\end{aligned}
$$

In the first contact phase, the friction force components have been shown as in Figure 6(b). The current external contact force (including input force and friction force) components which cause bending stress should be

$$
\begin{gathered}
f=F_{n} \times \mu, \\
F_{v}=F_{t}-f_{t}, \\
F_{h}=F_{r}+f_{r},
\end{gathered}
$$

where $\mu$ is the coefficient of friction.

In the second contact phase, the friction status is shown as in Figure 6(c), friction force direction is opposite to the first contact phase, and the current external contact force components which cause bending stress should be

$$
\begin{gathered}
f=F_{n} \times \mu, \\
F_{v}=F_{t}+f_{t}, \\
F_{h}=F_{r}-f_{r},
\end{gathered}
$$

where $\mu$ is coefficient of friction.

The formulas explain significant phenomena owing to sliding/rolling frictional effect. The friction force component counteracts the input force component in vertical, and the directions of horizontal contact force component and the elastic deformation are different, so the high horizontal contact force components will decrease bending stress. Therefore, the bending tensile stress should be smaller than nonfrictional model in the first phase. In the second contact phase, the friction force direction has become opposite, the friction component and input force component in vertical are in the same direction to amplify resultant contact force, and the horizontal input force component has also been weaken

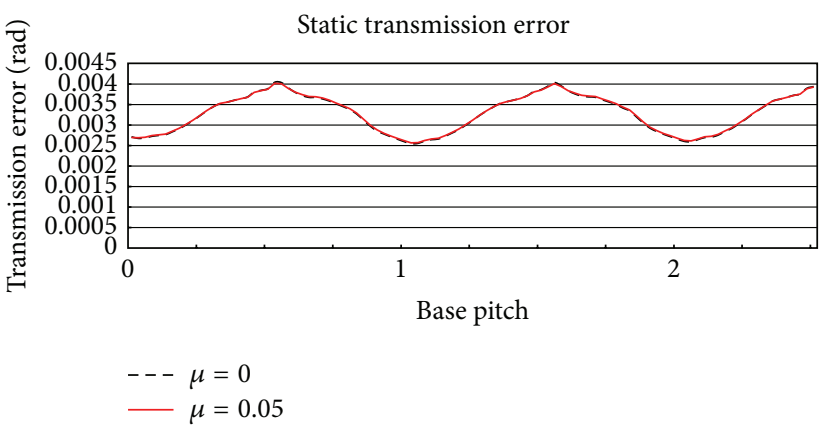

FIGURE 7: Static transmission error results.

due to friction force component. Consequently, it should be larger than the non-frictional model. In addition, the similar phenomena have been displayed by relevant further investigations regarding static bending shearing stress. In fact, the perfect work conditions are impossible for actual meshing process, the friction must exist between contacted surfaces, and so a conclusion regarding frictional effects on bending stress base on static analysis can be summarized like this: the bending stress of gear tooth will be amplified by frictional effect in the phase in which the contact point moves from start point to pitch point and will be weaken by frictional effect in the phase which the contact point moves from pitch point to end point.

Figure 7 is the result of gear static transmission error with static boundary conditions group. It reflects some significant phenomenon derived from frictional effect.

Some conclusions can be summarized according to the result figure. First, the segments of individual result line in each base pitch are the same. Second, generally speaking, transmission error is always considered as the main source of noise and vibration in gear meshing. The reason is that the transmission error leads to serious impact and extrusion between contacted gear teeth in meshing. The transmission error undulations in the result figure implicate that the gear must produce vibration in meshing due to the rotation displacement differences between pinion and wheel, and the vibration is the reason of referred impact and extrusion. 
Third, the result indicates that the frictional effect on static transmission error is very limited. There are no remarkable differences caused by friction. But in fact, the transmission error status can be influenced by lots of boundary conditions. The results of static analysis can only display the most typical situations of transmission error and the accuracy of static transmission error result is not very satisfied and reliable. The relevant investigations of dynamic analysis should be more accurate to reflect the actual situations.

To summarize, the frictional effect on static transmission error assuredly exists, but it is not very obvious and can be ignored completely.

\section{Dynamic Bending Stress and Transmission Error}

The introduction regarding dynamic research schemes also bases on the same contents as static analysis. In fact, the dynamic analysis is much more complicated than static analysis, and some significant phenomena which cannot be indicated in static analysis will be discussed carefully in dynamic analysis. Many of dynamic boundary conditions will lead obvious excitations to affect the characteristics of gear contact significantly. A basic standpoint of this thesis is that the transmission error is a main source of harmful noise and vibration, but strictly speaking, it is not the only reason of those nonlinear responses. The empirical researches have indicated that the vibrations and noise are all derived from various excitations of certain characteristic parameters of gear contact, and the transmission error is only one particular element of those excitations. It should consider that the vibration and noise in gear meshing are particular responses which are caused by various dynamic excitations, and the excitations can also lead some nonlinear responses to make the dynamic performance of meshing gears more complicated. So the significant objectives of dynamic analysis are to investigate some typical responses to dynamic excitations and to decrease harmful influences as effectively as possible.

The excitations include two categories mainly which are "external" and "internal" excitations, and the internal excitations are significant dynamic excitations of meshing gears. The external excitations are always caused by some external boundary conditions such as load, input moment and power, and engine speed; the particular responses are similar to other transmission systems. Internal excitations of gear set are dynamic excitations in gear meshing process, and it includes three basic types: stiffness excitation, transmission error excitation, and meshing impact excitation. However, all of the internal and external excitations must exist in actual meshing process and affect gear performance seriously. So the excitations make the actual situations of gear meshing more complicated and lead many problems in dynamic analysis. Although the finite element method can simulate the gear meshing process better and produce less error than other mathematical analytical methods, it is still hard to gain very accurate results and achieve perfect objectives.

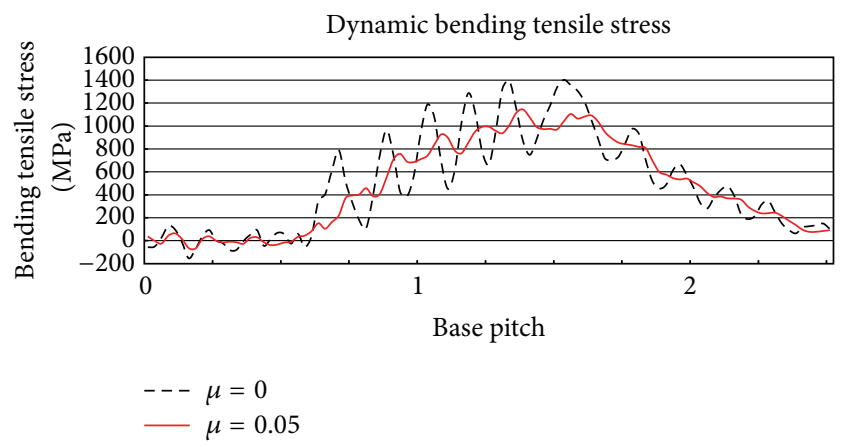

FIGURE 8: Dynamic bending tensile stress results ( $\omega=100 \mathrm{rad} / \mathrm{s})$.

It is also necessary to mention that the dynamic investigations in this research are only on the basis of some empirical conclusions and simple linear and nonlinear vibration theories, so the conclusions and discussions are not very accurate in nature. Furthermore, the research strategy can not cover all complicated dynamic responses caused by various external and internal excitations in gear meshing completely it is only hoped to display and discuss some basically typical phenomena which are derived from dynamic responses. Figure 8 displays the particular situation of dynamic bending tensile stress with the angular velocity being $100 \mathrm{rad} / \mathrm{s}$. It is far different from the results of static analysis.

There are serious fluctuations of each result line and the phenomenon implicates the internal excitations in gear meshing. But based on the results of static analysis as a reference, the general varying trend of dynamic bending stress is still similar to static bending stress, and it is easy to explain that the undulations of dynamic bending stress must derive from the internal excitations. The undulation is a kind of typical dynamic transient response, and according to the result figure, the internal excitations will not affect the steady responses very seriously.

The static bending stress results have indicated the difference between the relative positions of two tensile stress result lines in different contact phases due to frictional effect, and the interaction point of the two lines means the gear teeth are contacting at pitch point. It is an important and significant conclusion regarding frictional effect on static bending stress. In dynamic analysis, the situation is similar, but the general situation of relative positions of two result lines is not very obvious due to the undulations, and the significant interaction point is hard to display to implicate the moment when the teeth are contacting at pitch point. These particular phenomena are the most significant differences between the results of static and dynamic analysis.

Figure 9 is the dynamic transmission error result during 2 base pitches. To compare with the result of static analysis, the general varying trends of dynamic and static TE result lines $\mu=0.05$ are similar; it implicates that the steady response of dynamic transmission error also accords with the static rules. The undulations of dynamic transmission error lines derive from various dynamic excitations, and in particular, the vibrations of gear set and the impacts between contacting gear teeth are main sources. In addition, the steady response 


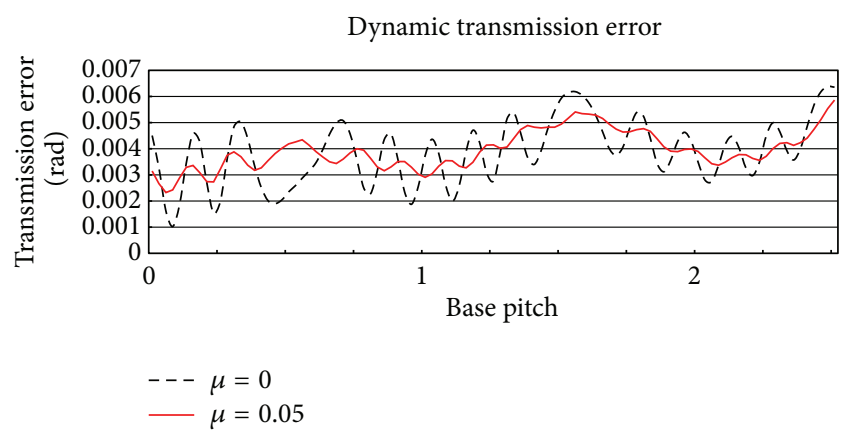

Figure 9: Dynamic transmission error results $(\omega=100 \mathrm{rad} / \mathrm{s})$.

of dynamic TE result lines $\mu=0.05$ line in the first base pitch does not obviously accord with the basic static rules. It is believed that the reason of this situation is the gear's inertia during the wheel gear run from quiet to unit rotary speed, so the steady response during this period is not obvious due to the wheel gear's inertia.

According to the result lines in Figure 9, the frictional effects on dynamic transmission error can be indicated. In static analysis, the friction will not affect transmission error significantly, but the frictional effect on dynamic transmission error has been amplified seriously and could not be ignored any more. Figure 9 shows the line $\mu=0.05$ is little more steady and regular than the line $\mu=0$. This phenomenon implicates that the friction has affected both the steady and transient responses by decreasing the amplitude of undulations. Generally speaking, the difference between the maximum and minimum peak values of transmission error is the main reference to estimate the noise intensity roughly; the situation of transmission error results in Figure 9 implicates that the frictional effect can decrease gear noise obviously due to dynamic excitations.

\section{Summary}

The paper analyzed the frictional effects on bending tensile stress and transmission error in static and dynamic boundary conditions. Base on the discussions and explanations in this paper, the integrated frictional effects can be summarized. The frictional effects on bending tensile stress are listed as follows.

(1) The friction can decrease bending stress during the first contact phase, but the bending stress will be increased due to friction during the second contact phase.

(2) The moment when the frictional shear stress disappears implicates that the gear teeth are contacting at pitch point, and the frictional effects will become opposite from now on.

(3) The degree of frictional effects on bending stress depends on the coefficient of friction of contact surface and input torque.
(4) Dynamic frictional effects on bending stress are similar to static frictional effects. But the dynamic frictional effects are not very obvious, and the moment when the friction shear stress disappears is hard to be indicated due to the various dynamic excitations.

(5) Friction can weaken the bending stress undulations (typical transient response) due to various dynamic excitations.

The frictional effects on transmission error should be concluded as follows.

(1) In static analysis, the friction can increase result values, but the noise will be decreased slightly due to reliable friction.

(2) In dynamic analysis, the friction can decrease harmful responses to dynamic excitations. The transient responses will also be weakened, and the transmission error result line will become more steady and regular.

(3) Friction can also affect noise in dynamic analysis, but it is believed that too serious friction can make the transmission error result line more complicated.

(4) Dynamic frictional effects on transmission error are not steady, and it will be affected and changed gradually during the gear meshing.

(5) The steady responses will not be changed significantly due to friction.

The results of research indicate that the frictional effect is an important boundary condition in gear design. For gear static performance, friction will affect the bending stress obviously; it should be considered carefully in gear tooth strength analysis. For dynamic performance, although the tooth profile modification can regulate complicated transmission error, the frictional effect will affect modification scheme in high load work conditions. To sum up, friction effect should not be ignored; it will not only affect static stress and transmission but also produce other boundary conditions which may affect gear performance; for example, the sliding friction will produce thermal in meshing, and there is a little difference of the tooth profile curve by thermal effect, and the profile modification scheme should consider it to regulate. In actual manufacture processes, the frictional effect will affect production performance seriously; designers should pay more attention to avoid or use frictional effect for optimizing gear performance.

\section{References}

[1] M. A. Barakat, "Determination of instantaneous coefficient of gear tooth friction," Journal of Engineering Sciences, vol. 8, no. 1, pp. 9-19, 1982.

[2] A. Ishibashi, S. Ezoe, and S. Tanaka, "Friction coefficients between contacting teeth at start of running," pp. 845-850, Institution of Mechanical Engineers, Proceedings of the IMechE Conference.

[3] S. Vijayarangan and N. Ganesan, "Static contact stress analysis of a spur gear tooth using the finite element method, including 
frictional effects," Computers and Structures, vol. 51, no. 6, pp. 765-770, 1994.

[4] B. Rebbechi, F. B. Oswald, and D. P. Townsend, Measurement of Gear Tooth Dynamic Friction, vol. 88, American Society of Mechanical Engineers, Design Engineering Division, 1996.

[5] P. Velex and P. Sainsot, "An analytical study of tooth friction excitations in errorless spur and helical gears," Mechanism and Machine Theory, vol. 37, no. 7, pp. 641-658, 2002.

[6] A. Mihalidis, V. Bakolas, K. Panagiotidis, and N. Drivakos, "Prediction of the friction coefficient of spur gear pairs," VDI Berichte, vol. 2, no. 1665, pp. 705-719, 2002.

[7] S. Kim and R. Singh, "Gear surface roughness induced noise prediction based on a linear time-varying model with sliding friction," JVC/Journal of Vibration and Control, vol. 13, no. 7, pp. 1045-1063, 2007.

[8] S. He, R. Gunda, and R. Singh, "Inclusion of sliding friction in contact dynamics model for helical gears," Journal of Mechanical Design, vol. 129, no. 1, pp. 48-57, 2007. 

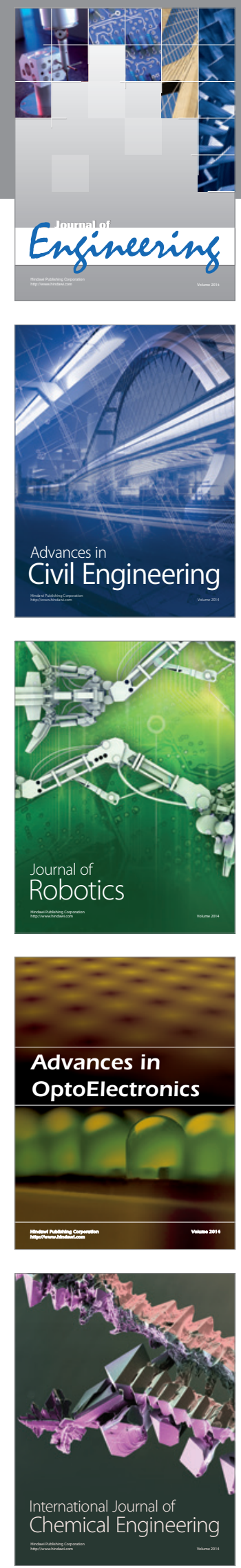

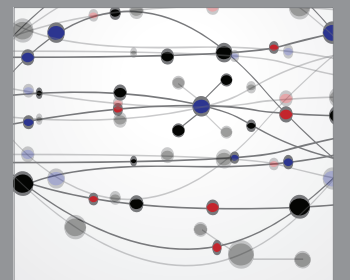

The Scientific World Journal
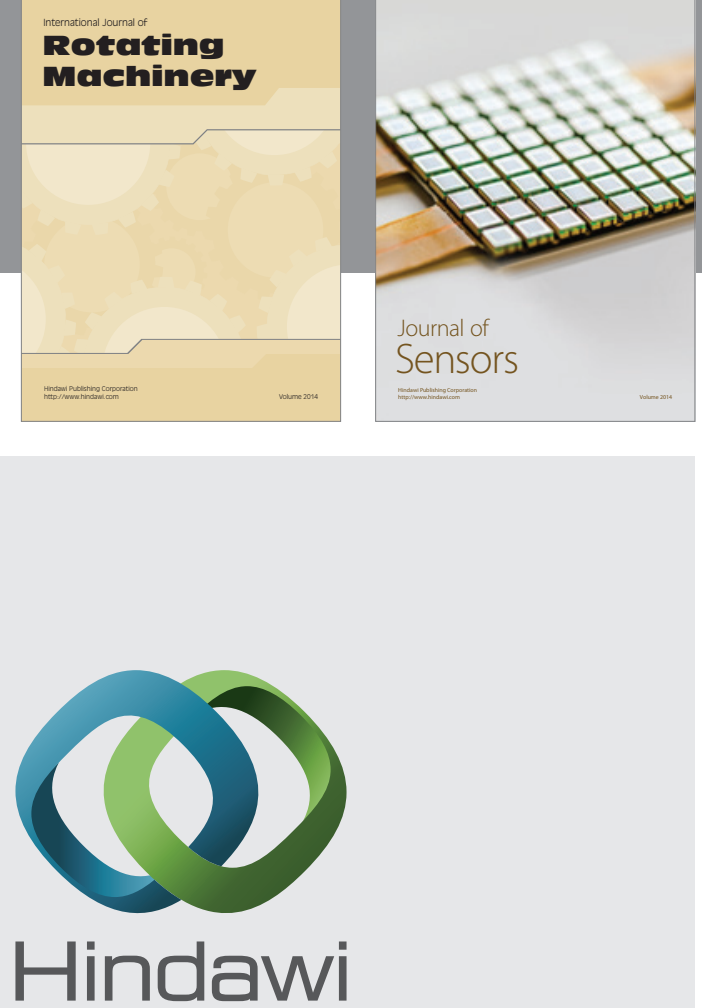

Submit your manuscripts at http://www.hindawi.com
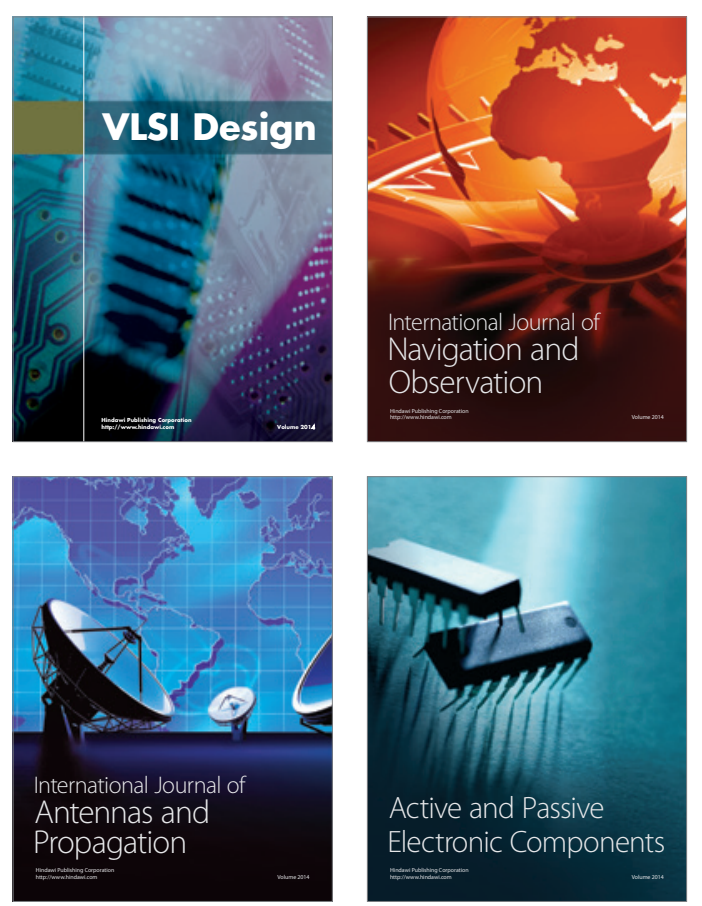
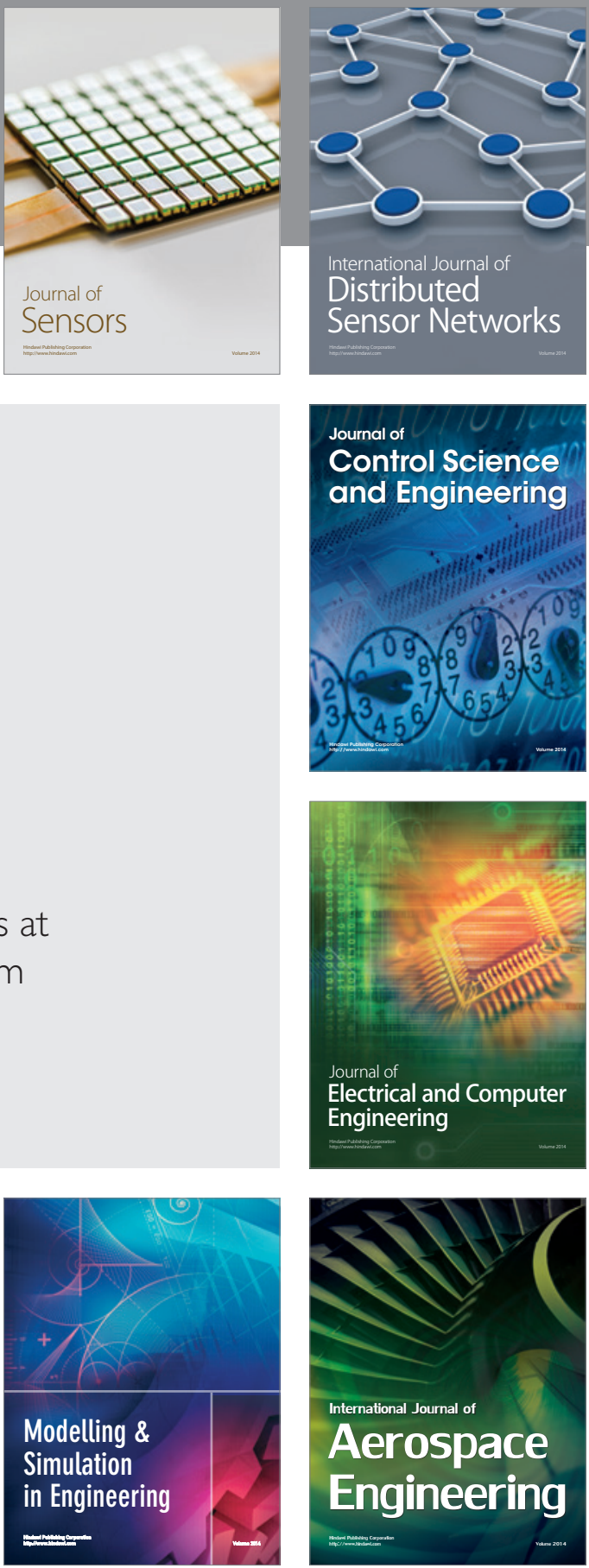

Journal of

Control Science

and Engineering
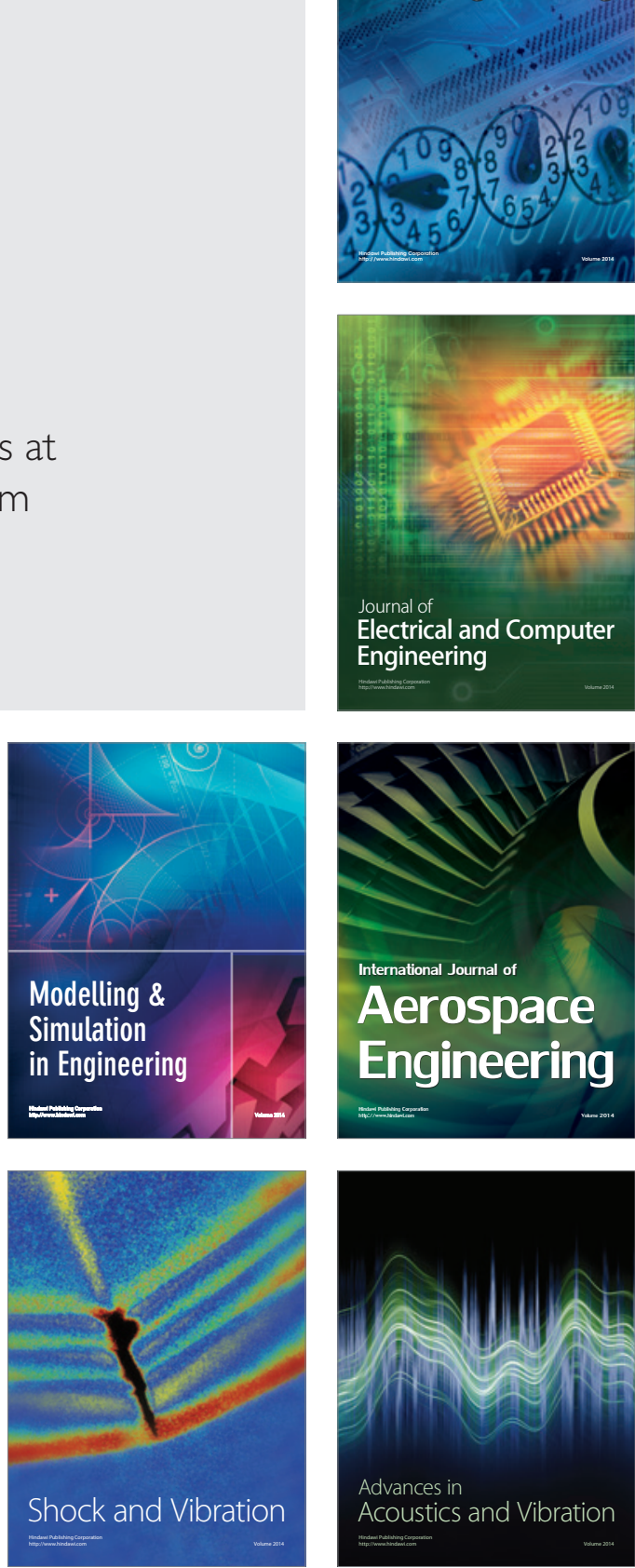